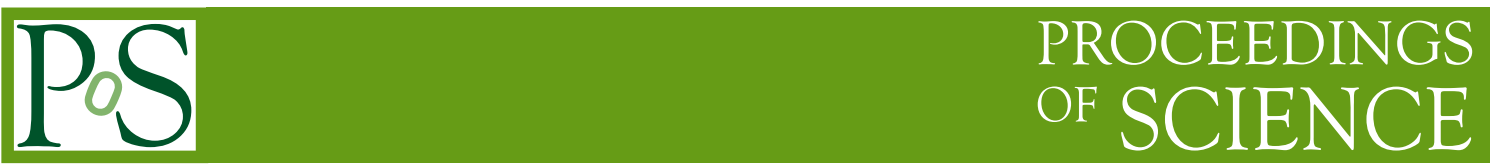

\title{
Astroparticles in Latin America: current status and outlook
}

\section{Ivan Sidelnik*}

Centro Atómico Bariloche, CNEA, CONICET and Instituto Balseiro, Av. Bustillo km 9.5, San

Carlos de Bariloche, Argentina

E-mail: sidelnikecnea.gov.ar

The successful installation and operation of the Pierre Auger Observatory in Argentina has been a milestone in Astroparticle research in Latin America, generating new regional research opportunities in the field. In this context, the LAGO project, begun in 2005 with the aim of studying the high-energy component of gamma ray bursts (GRBs). This observatory consists of different arrays of water-Cherenkov detectors installed in high altitude mountains throughout Latin America. Recently, it has demonstrated the feasibility of conducting studies on the solar modulation of the galactic cosmic ray flux. Currently more than 80 scientists and students from Mexico, Guatemala, Colombia, Venezuela, Ecuador, Peru, Bolivia and Argentina are integrated into the LAGO Collaboration. The high level of regional integration in the scientific community reached thanks to this kind of major projects, has led to the recent formation of the CLES (Consorcio Latinoamericano de Experimentos Subterráneos). This organization promotes the creation and installation of the ANDES Underground Laboratory to be built inside the projected International Agua Negra tunnel between Argentina and Chile. The ANDES laboratory with over 1750 meters of rock cover, will be the first laboratory of its kind to be installed in the Southern Hemisphere.

10th Latin American Symposium on Nuclear Physics and Applications

1-6 December, 2013

Montevideo, Uruguay

\footnotetext{
*Speaker.
} 


\section{Introduction}

The study of astroparticle physics has a long tradition in the Latin American region. After the discovery of cosmic radiation by Victor Hess in 1912, it was understood that information about the universe is arriving to the earth not just in the form of light, but also in particles with mass. Astroparticle physics studies particle interactions by using astrophysical sources. It also studies the properties of those objects through the particles that we measure at Earth.

In Latin America the story probably starts in the late forties with the discovery of the mesons by Lattes, Occhialini and Powell in the Chacaltaya mountains, in Bolivia [1]. This meson happens to be the pion, and this discovery was made thanks to the altitude that Chacaltaya mountains provide, to measure particles that weren't attenuated by the atmosphere, and the improvements that Lattes made in the emulsion of the photographic plates. This led to a nobel prize awarded to Cecil Powell. Another experience in the study of cosmic rays was carried out by undergraduate students and researchers from Argentina, on the Aconcagua mountain [2]. In the early fifties, using photographic emulsions they travelled to different sites in the Andes mountains range and measured the track of particles at different altitudes and latitudes studying the effect of this on cosmic rays. These first experiences gave birth to facilities, institutes and open divisions at different Universities around Latin America that started to focus on the study of cosmic ray, nuclear and high energy particle physics.

This paper intends to give a brief review of some of the largest ground based observatories that are running nowadays measuring the properties of cosmic rays and gamma-ray bursts, and their application to space weather studies. The Pierre Auger Observatory, the largest cosmic ray detector in the world, located in Argentina, is described with its latest results in section 2. Section 3 and 4 describe facilities that were conceived in principle, as gamma-ray bursts observatories, but they can do studies of space weather phenomena and cosmic ray also: LAGO (that spans over all Latin America from Mexico to Antarctica) and HAWC, in Mexico, respectively. There is a new project that intends to measure solar transient events through the study of gamma-ray burst and cosmic ray up to the knee energies, called PAS, section 5. The last facility described is ANDES, an underground laboratory that will be built in a tunnel that goes through the Andes mountains between Argentina and Chile, section 6. The final remarks are given in section 7.

\section{The Pierre Auger Observatory}

The Pierre Auger Observatory is a collaboration of more than 500 members coming from 18 different countries. Is located in Malargüe, at the south of the Province of Mendoza, Argentina (1400 m a.s.l.). Its main goals are studies of the Ultra High Energy Cosmic Rays (UHECR). The Observatory includes different instruments working all together with a unique design: it was conceived as a hybrid detector combining two different techniques of measuring UHECR. The surface detector array (SD) is composed of 1660 water-Cherenkov detectors (WCD) placed on a triangular grid of $1500 \mathrm{~m}$ covering $3000 \mathrm{~km}^{2}$. Each station is filled with 12 tons of water and instrumented with three photomultiplier tubes (PMTs) which detect the Cherenkov light produced by the charged particles that go through the water [3]. On the other hand, the fluorescence detector (FD) comprises 24 optical telescopes grouped in four sites, observing the atmosphere above the 

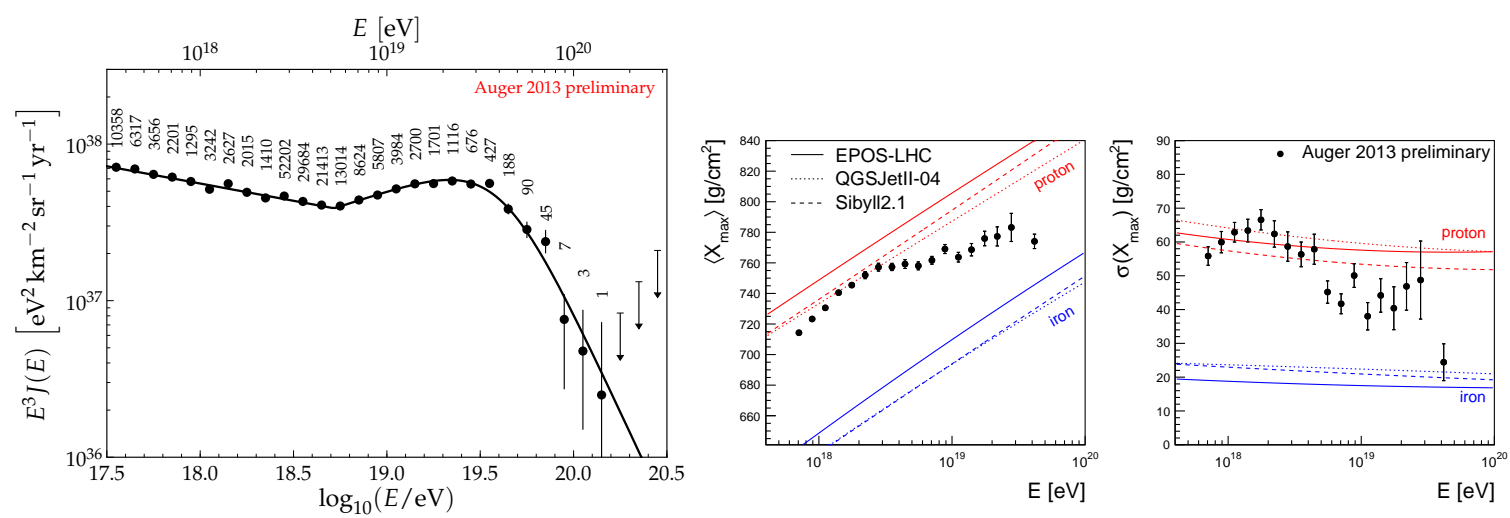

Figure 1: Left: Combined energy spectrum of UHECR measured by the Pierre Auger Observatory. The numbers gives the total events in each energy bin. Right: change in the mass composition of UHECR as measured by the Auger observatory, using the mean value and the fluctuation of the $\mathrm{X}_{\text {max }}$ compared to the prediction of different hadronic models [6].

array. Each telescope consists of an $11 \mathrm{~m}^{2}$ segmented spherical mirror that focuses the fluorescence light into a camera composed of 440 PMTs, that operates during moonless night [4]. The synergy between the SD and FD makes possible the calibration of the energy of SD events. This has an almost $100 \%$ duty cycle, using the calorimetric energy measured by the FD ( $\sim 10 \%$ duty cycle).

There are upgrades being carried out in the observatory towards low energy cosmic ray measurements. A denser surface array called "the infill" [6], composed of 71 WCD with spacings of $750 \mathrm{~m}$ each, covering an area of $\sim 30, / \mathrm{m}^{2}$ it was installed and has been running since 2008. This array enables the capability of measure cosmic rays with energies from $3 \times 10^{17} \mathrm{eV} \mathrm{[5],} \mathrm{extending} \mathrm{the}$ dynamic range of the observatory to 3 orders of magnitude in energy. This is part of the AMIGA extension that will also have buried muon counters to measure the chemical composition of cosmic rays by measuring the muon content of the shower in a independently. Nowadays there are seven of these detectors installed in the field [6]. There is also a FD upgrade called "High Elevation Auger Telescopes" (HEAT) that looks over the infill site with three telescopes, maintaining the hybrid concept of the detector.

Since 2004 the Pierre Auger Observatory has detected high quality UHECR events, performing key measurements of the energy spectrum, mass composition and anisotropy of this cosmic rays. In spite of that there are many issues still not understood.

In Fig. 1, left is shown the combined spectrum of the observatory using SD only, hybrid, inclined and infill measurements. It can be seen to span over three orders of magnitude in energy [6]. This measurement shows that the suppression and the ankle are very well established. But despite its high statistical accuracy, the energy spectrum alone is not enough to distinguish between the different astrophysical scenarios that can explain these features. There are too many unknowns like the source distributions and evolution, the acceleration characteristics, and the mass composition. That is why this kind of measurements has to be combined with anisotropy and mass composition measurements.

The results regarding the $\left\langle\mathrm{X}_{\max }>\right.$ and $\sigma_{X_{\max }}{ }^{1}$ and their evolution in energy are shown in Fig.

\footnotetext{
${ }^{1}$ These are the two first moments of the $\mathrm{X}_{\max }$ distribution, the position of the maximum shower size.
} 

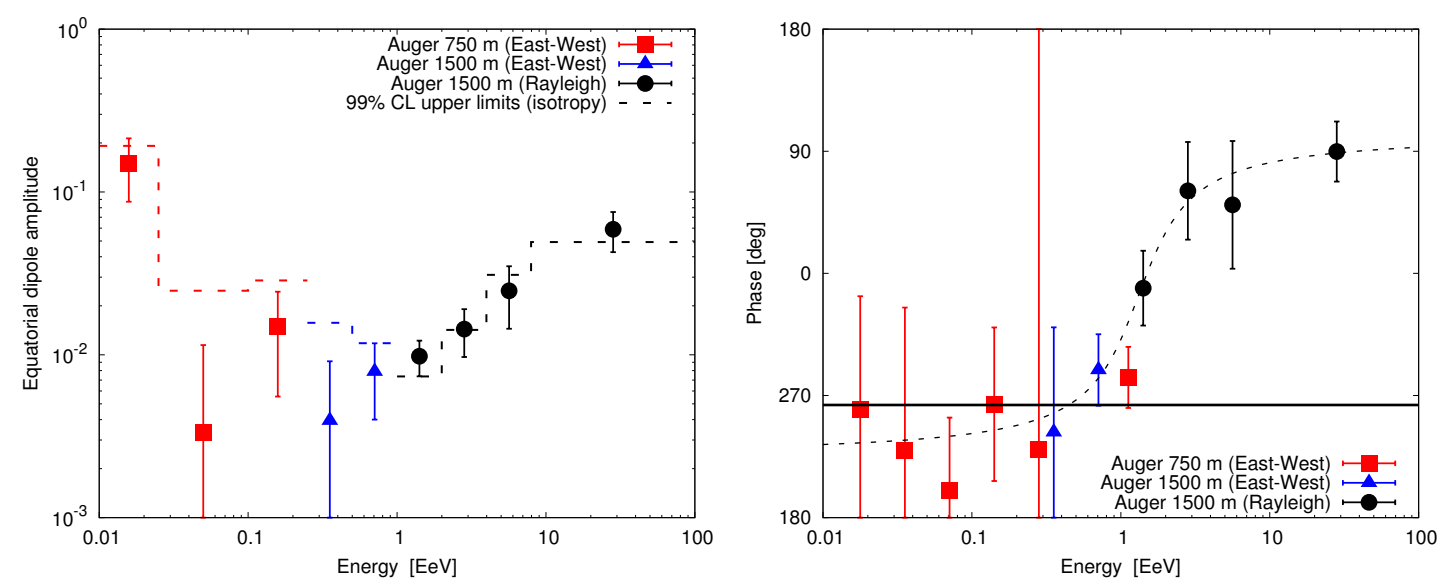

Figure 2: Left: Equatorial dipole amplitude as a function of energy. The dashed lines are the 99\% CL upper values of the amplitude that could result from fluctuations of an isotropic distribution. Right: phase of the first harmonic as a function of energy, it can be seen that there is a phase transition from the galactic centre towards the anti-galactic centre directions and an alignment at low energies that could be a hint of large scale anisotropy [6].

1 , right. When they are compared to different hadronic models, the data seems to change the behaviour above the ankle region $(1 \mathrm{EeV})$.

There has also been an analyses on the arrival direction of cosmic rays performed over the extended energy range, studying the first harmonic modulation in right ascension. Fig. 2, left, shows the amplitude of the equatorial dipole that, thanks to the East-West method, spans over four orders of magnitude in energy. While there is no clear evidence of anisotropy, it is worth noting how, above $1 \mathrm{EeV}, 3$ of the 4 points are above the $99 \% \mathrm{CL}$. On the other hand the phase evolution, Fig. 2, right, shows an interesting trend with a smooth transition from the galactic centre direction towards the galactic anti-centre. To test this hypothesis of a change in the phase there has been set an analyses of an independent data set, such that when a certain number of events is reached (by the beginning of 2015) this data set will be ready to confirm or reject the veracity of this result. A summary of all this contribution and details is available in [7].

\section{Large Aperture Gamma ray burst Observatory (LAGO)}

Gamma-ray bursts (GRBs) are the most powerful explosions known in the Universe. They are sudden emissions of gamma-rays lasting very short time intervals (from 0.1 up to 500 seconds). They were discovered in 1967 by the Vela satellites that the US government launched to monitor covert nuclear tests from space. In 1997 the BeppoSAX satellite detected an optical afterglow of a GRB. When it was analyzed it showed that the redshift of the galaxy that originated the GRB was at more than 6 billon light years from Earth. This showed GRBs to be extragalactic events. The astrophysical sources of these events are still unclear but good candidates are the coalescence of compacts objects for the short bursts (less than $2 \mathrm{~s}$ ), and hypernovae, supernovae produced by very massive stars, for the long bursts (more than $2 \mathrm{~s}$ ) [9].

Despite satellites observations like SWIFT, FERMI and others, revealing some questions about the origin and location of GRBs, other questions remain unanswered, especially in the high energy 

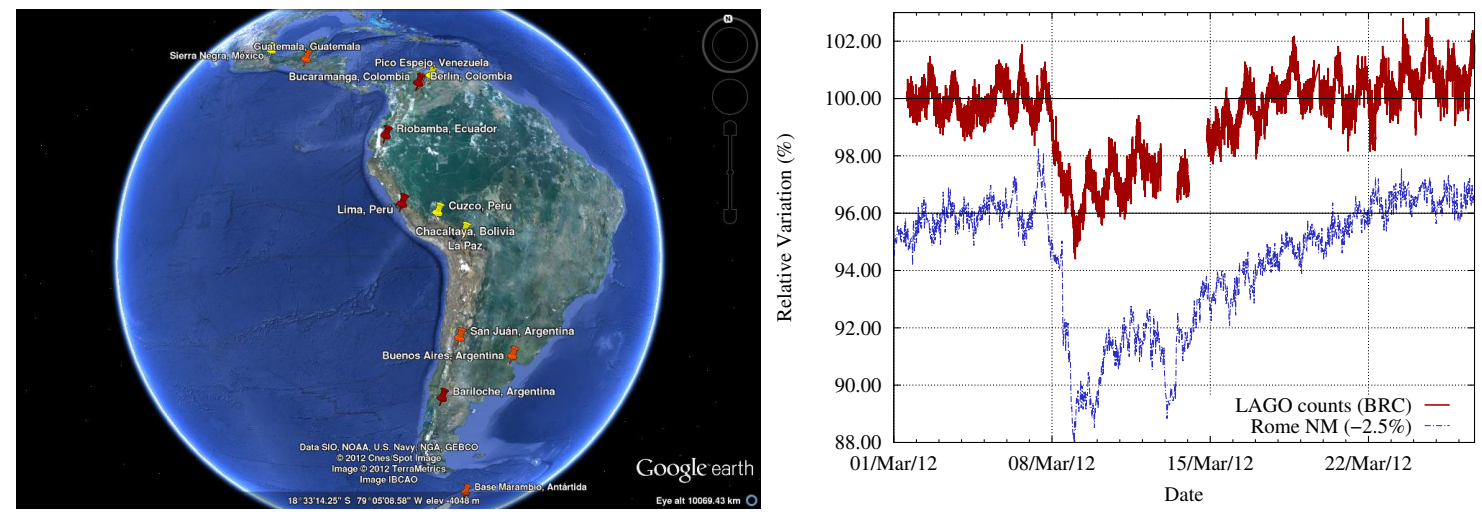

Figure 3: Left: Google earth map showing all LAGO sites. For the color code see text. Right: a Forbush decrease event seen by a single LAGO detector. The comparison between the WCD in Bariloche and a neutron monitor in Rome shows a very good agreement [14].

region, i.e. the spectrum. Up to now no ground-based experiment has detected a GRB.

The LAGO project is a recent collaboration that comes from the association of latin american astroparticle researchers. It started in 2005 and it was designed to survey the high-energy component of GRBs. It is a network of ground-based WCDs, located at mountain altitudes, above 4500 m.a.s.l., where the flux of the primary gamma-rays is too low for effective detection by small area satellite detectors. This collaboration was motivated by the experience of the Pierre Auger Observatory, and the idea is to install uchhis WCD in 9 latin american countries: Argentina, Bolivia, Brasil, Colombia, Ecuador, Guatemala, Mexico, Peru and Venezuela [10, 11]. More than 75 people integrates the LAGO community, keeping a close collaboration with researchers from IN2P3 of France and INFN in Italy.

The idea is that when high energy photons from GRBs reach the atmosphere, they produce cascades that can be detectable at ground level by using WCDs. Instead of trying to detect this extensive air showers, LAGO makes use of the single particle technique, that is to observe an excess in the counting rates of secondary particles produced in EAS [12]. The main advantage of using WCDs compared to other instuments, is their higher sensitivity to photons, which are $90 \%$ of the secondary particles at ground level for high energy primary photons.

Although the original plan comprised the detection of the high energy component of GRBs, recently it has been shown that WCDs can also be used to study the Solar Modulation (SM) of galactic cosmic rays and other transient effects, by measuring the variations of the flux of secondary particles at ground level [13].

The main effect is produced by the solar magnetic field. When the Sun shows a high activity (intense magnetic field), galactic cosmic rays are deflected, resulting in a reduction of their flux on Earth. When magnetic fields are less intense we have a higher flux. So, by measuring the flux of galactic cosmic rays one can determine indirectly the solar activity. This allows, for instance, the observation of the eleven year cycle of the sun and also transient events called Forbush decreases. These kind of events are produced when a Coronal Mass Ejection (CME) is originated in the Sun, resulting in a huge mass of plasma sent through the interplanetary medium. Upon reaching Earth, this plasma perturbs the near space and results in a modification in the flux of galactic cosmic rays. 
A rapid diminution of their flux can be observed (a few percent in a few hours). Then, once the CME goes on his way, the galactic cosmic ray flux slowly comes back to its original value, on a time scale of days. This reduction in the flux is called Forbush decrease.

LAGO can be an optimal detection network to characterize the SM and transient events, as it spans over a large area with sites at different latitudes, longitudes and geomagnetic rigidity cut offs.

Recently the LAGO Observatory consists of different sites, in Fig.3, left, a map of the Latin American region can be seen with the places where a LAGO WCD is in operation or to be installed: Sierra Negra in Mexico at $4550 \mathrm{~m}$ a.s.l. was the first LAGO site and has been in operation since 2007, it has three $2 \mathrm{~m}^{2}$ and two $1 \mathrm{~m}^{2}$ WCD. Chacaltaya in Bolivia at $5250 \mathrm{~m}$ a.s.l. is the highest site of LAGO, and has three $4 \mathrm{~m}^{2}$ and two of $1 \mathrm{~m}^{2}$ WCD. They have been taking data since 2008. Also there is one in Cusco, Peru at $4450 \mathrm{~m}$ a.s.1., with one $2 \mathrm{~m}^{2}$ WCD taking data since 2010. There is another WCD in Bariloche, Argentina, a prototype that was used for calibration and software tests since 2006, but now has shown that an analyses of SM can be done. Fig. 3, right shows the comparison between a neutron monitor in Rome, and a single WCD of $1.8 \mathrm{~m}^{2}$ in Bariloche [14] measuring a Forbush decrease in 2012. As can be seen the LAGO detector shows similar relative variation as the other two measurements.

Various other WCDs are installed or being installed: Huancayo and Lima, Peru, Buenos Aires, Argentina, Cochabamba, Bolivia, and Riobamba, Ecuador are in the process of installation. Also the cities of Guatemala in Guatemala, Chimborazo in Ecuador, San Juan with the Marambio base in Artantica (Argentina) are planned to host WCDs [15]. There is also a plan to include Brazil [16]

\section{The High Altitude Water Cherenkov Observatory (HAWC)}

HAWC is a survey instrument that aims to study gamma-ray sources in the $\mathrm{TeV}$ region and it is the successor of the Milagro observatory that was located in New Mexico[17]. It is a joint collaboration between a large number of USA and Mexican universities and scientific institutions, including UNAM, Los Alamos National Laboratory and the NASA/Goddard Space Flight Center. The construction of HAWC started in 2011. It is located at $4100 \mathrm{~m}$ a.s.l. in Mexico, near the Sierra Negra Volcano close to the city of Puebla. It uses WCDs allowing the observation of cosmic rays also. Each Cherenkov detector consists of $180 \mathrm{~m}^{3}$ of extra pure water inside an corrugated steel tank of $5 \mathrm{~m}$ high and $7.3 \mathrm{~m}$ of diameter with four PMTs fixed to the bottom of the detector. Currently one third of the observatory is in operation and taking data.

The HAWC observatory will have an order of magnitude better sensitivity, angular resolution, and background rejection than the Milagro experiment. The improved capabilities will allow the detection of both transient and steady emissions, the study of the Galactic diffuse emission at $\mathrm{TeV}$ energies, and it will measure or constrain the $\mathrm{TeV}$ spectra of $\mathrm{GeV}$ gamma ray sources. Also, it will be capable of detecting prompt emission from gamma ray bursts above $50 \mathrm{GeV}$. A review of this observatory with the status and latest results can be found in [17].

\section{Polo de Astronomía Social (PAS)}

Due to its geographic characteristics, the Andinian Páramo located near Berlín, Colombia, at 
$3500 \mathrm{~m}$ a.s.l., is an excellent location to build an array of particle detectors to study cosmic rays in a wide energy range, including the solar activity modulation of cosmic rays, gamma-ray bursts (GRB), and the high energy region of the cosmic ray spectrum. The proposed array will consist of more than one hundred autonomous and wireless WCDs located over different concentric triangular grids with different spacing between neighbor detectors, spanning over a total area of more than $16 \mathrm{~km}^{2}$. This facility will be operated by an interdisciplinary group of researchers closely related to the LAGO international Collaboration.

The design of the detector array is based on Corsika air shower and Geant4 based detector response simulations. The proposed design of this array will allow one to implement two different measurement modes: the counting mode and the shower mode. In the counting mode, the variations in the recorded flux of secondary particles at detector level can be correlated with transient phenomena, such as the solar modulation of galactic cosmic rays or the arrival of the highest energy component of an energetic GRB; or long term flux modulations related with the solar activity cycle. In the shower mode, in contrast, there will be a search for time-space correlated signals in different, non-aligned, detectors of the array. In this way, it will be possible to determine the main parameters that characterize the extensive air shower produced by the interaction of a single high energy cosmic ray with the atmosphere. From this parameters, the arrival direction and the energy of the impinging cosmic ray will be obtained. The size of the array and the increasing spacing between detectors will allow one to complement present measurements in the so called knee region of the cosmic ray energy spectrum $\left(E \sim 10^{15} \mathrm{eV}\right)$ and beyond [18].

\section{Agua Negra Deep Experiment Site (ANDES)}

In the line of big astroparticle experiments that were built in Latin America during the pasts years, a new facility is being developed. Deep underground laboratories are one of the principal contributors to astroparticle physics. In the past sixty years they provided unique ways of studying weakly interactive particles. Nowadays more than a dozen underground laboratories are running or being constructed, studying subjects such as low radioactivity background measurements, neutrino physics or dark matter searches. All of these laboratories are located in the northern hemisphere. A review of underground facilities around the world can be found in [19].

In the past, some attempts were made to install underground laboratories in the southern hemisphere, but none of them were maintained. There was one in a gold mine in South Africa that contributed to the discovery of atmospheric neutrinos in 1965, another installed in an iron mine in Argentina in 1995 to seek for dark matter oscillation signals, and also there were unsuccessfully searches performed for mines in Brazil and Chile, see [20] and references in there.

A demand for a southern hemisphere laboratory has been growing since different experiments have claimed that they observed dark matter signals. DAMA/LIBRA has claimed the observation of a yearly modulation of their signals and attributed it to dark matter. To understand if this modulation is authentic, an experiment in the southern hemisphere observing the same kind of modulation is needed. If an opposed modulation is measured, this could indicate that the signal is coming from an atmospheric effect.

The Andes mountains are a natural limit between Argentina and Chile and have become of strategic economic importance in the region. The Agua Negra tunnel is the principal project that 


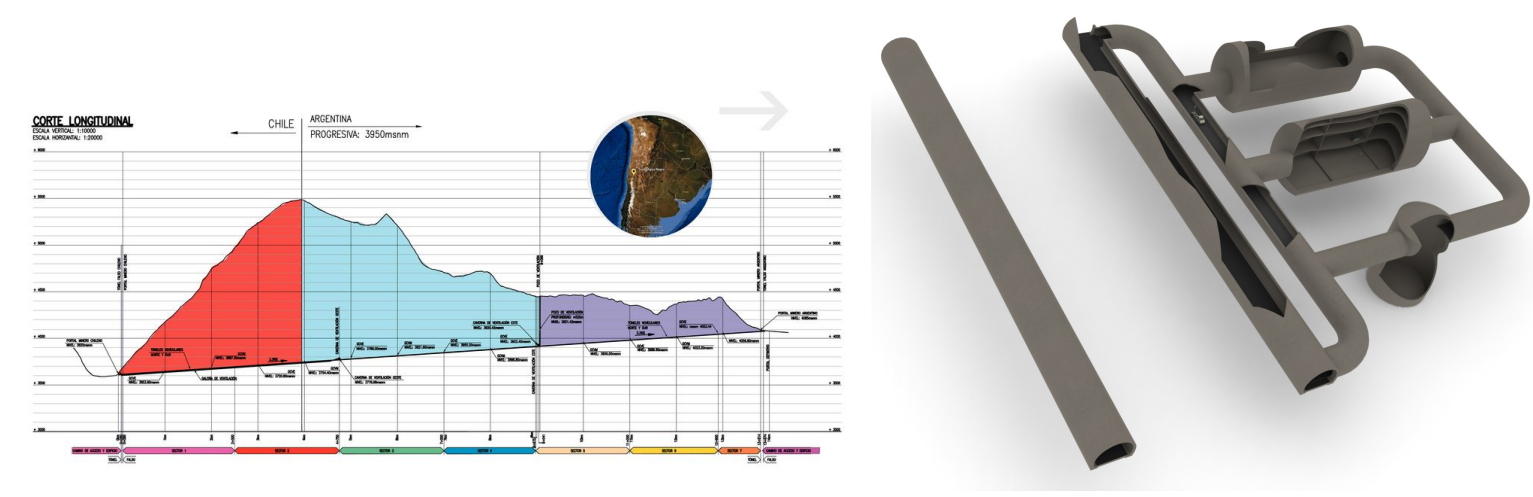

Figure 4: Left: Longitudinal cut of the Agua Negra tunnel and its location. Right: conceptual view of the ANDES deep underground laboratory, it will be located around $4 \mathrm{~km}$ inside the Agua Negra tunnel.

could improve the connectivity between Argentina and Chile. In 2012 the final project for this tunnel was proposed, and it is planned to be constructed between San Juan and Coquimbo. Also in March 2012 the presidents of Argentina and Chile gave the green light for the public tender. This process started in January 2013, and the international call for companies to compete in the tender was issued in June 2013. The tender process will last up to end 2014, given the difficulties of the bi-national civil work. The construction of the tunnel should start in 2015, and last for 7 years.

The tunnel design consists of double $14 \mathrm{~km}$ long road tunnel, each $12 \mathrm{~m}$ of diameter, separated by $60 \mathrm{~m}$. The entry points are at high altitude, $4100 \mathrm{~m}$ above sea level on the Argentine side, 3600 $\mathrm{m}$ on the Chilean side. Most of the ventilation for the tunnel is natural, thanks to the slope, with a forced ventilation system used in case of emergencies. The deepest point of the tunnel is located below the international limit between both countries, at about $4 \mathrm{~km}$ from the Chilean entry. With $1750 \mathrm{~m}$ of rock overburden, this spot is ideal to host a deep underground laboratory. Fig. 4, left, shows a cut in the Andes mountains were the tunnel is going to be constructed.

Given the relatively high altitude of the laboratory and its international location, two support laboratories are planned for ANDES. The Argentine laboratory is expected to be in Rodeo, a small town at $60 \mathrm{~km}$ from the tunnel entrance. It will be the closest support laboratory and be mostly used for day to day activities and the running of the experiments in ANDES. The Chilean laboratory will be located in La Serena, at $180 \mathrm{~km}$ from ANDES but in an internationally connected city, with strong scientific presence (such as the ESO for example). It will be mostly used for the preparation of the installation of the experiments and their testing.

The ANDES underground laboratory itself is foreseen to have a main hall of $21 \mathrm{~m}$ width, 23 $\mathrm{m}$ high and $50 \mathrm{~m}$ long, to host large experiments, and a big pit of $30 \mathrm{~m}$ of diameter and $30 \mathrm{~m}$ of height for a single large neutrino experiment. A secondary cavern of $16 \mathrm{~m}$ by $14 \mathrm{~m}$ by $40 \mathrm{~m}$ will host smaller experiments and services, while three smaller caverns ( $9 \mathrm{~m}$ by $6 \mathrm{~m}$ by $15 \mathrm{~m}$ ) will have dedicated experiments and a $9 \mathrm{~m}$ diameter by $9 \mathrm{~m}$ height pit will focus on low radiation measurements. A conceptual layout of the laboratory can be seen in Fig. 4, right.

The scientific programme of ANDES includes the main topics in astroparticle physics as neutrino and dark matter. There will be also a low radiation measurement laboratory, a geophysics laboratory, space for biology experiments, and possibly a particle accelerator to do nuclear astro- 
physics. In neutrino physics, different experiments will be run. The idea is that ANDES could host part of a large double beta decay experiment such as SuperNEMO. The flag experiment of ANDES will be a large neutrino detector similar to KamLAND and Borexino, but at in a 3 kton scale [21], focusing on low energy neutrinos [20]. This detector would allow complementary observation of neutrinos from a nearby supernova, something essential to properly study the effect of matter on neutrino oscillations. It will also be a geoneutrino observatory.

ANDES was considered from the beginning as a unique opportunity not only to build an underground laboratory for the international community but to build directly an international laboratory. Given its location on the borderline between two countries, and the current geopolitical unity displayed by Latin American countries, ANDES was proposed to be run by a consortium of Latin American countries, the CLES (initials of Latin American Consortium for Underground Experiments in Spanish or Portuguese). The CLES is currently formed by Argentina, Brazil, Chile and Mexico, and is foreseen to be open to more countries. It will be the organ in charge of the installation and operation of the ANDES deep underground laboratory and its support laboratories. It will also organise the academic integration of the scientific activities in the laboratory with the regional systems. The CLES should be a pole for underground science in the region [20, 22].

\section{Conclusions}

The lasts 10 years have seen the advent of different kind of ground based experiments to study astroparticle physics in the Latin American region. The Pierre Auger observatory has proved to be a very good role model of a scientific collaboration, with very important results in the ultra high energy cosmic ray field that nevertheless has left so far many unanswered questions. Many efforts are being made to upgrade the observatory to enlarge the capability of measure cosmic rays at lower energy. Also, this collaboration has given the experience to spin off experiments like LAGO that will be a large network spanning all over Latin America to measure GRBs and transient solar events. The installation of HAWC in Mexico has shown a very good starting and the PAS project a promising future.

One of the most important things is the future coming of the ANDES underground laboratory, a unique facility in the southern hemisphere, that will bring the opportunity to perform different kind of experiments from dark matter modulation to supernovae neutrinos and geoneutrinos. The construction is hoped to start in 2015 and it is foreseen as an international laboratory that can host experiments from all over the world coordinated by the CLES. Its large size and important depth will make it competitive with all existing laboratories.

\section{Acknowledgments}

I would like to thank the organizers of the $10^{\text {th }}$ LASNPA for allow me to give this invited talk and my colleagues of the Pierre Auger Collaboration for all this years I've learned so much. I also thanks Xavier Bertou and Hernán Asorey for their advice, the discussions and the review of this manuscript. Financial support by the Consejo Nacional de Investigaciones Científicas y Técnicas (CONICET) and the ITeDA institute via FUNC is also acknowledged. 


\section{References}

[1] C. M. G. Lattes, H. Muirhead, G. P. S. Occhialini and C. F. Powell Nature Processes involving charged mesons 159, 694-697 (24 May 1947) [doi:10.1038/159694a0]

[2] J. G. Roederer, Early cosmic-ray research in Argentina Physics Today 56(1), 32 (2003) [doi:10.1063/1.1554134]

[3] J. Abraham et al., Properties and performance of the prototype instrument for the Pierre Auger Observatory, NIM A 523 (1-2) 50-95, 2004

[4] J. Abraham et al., The fluorescence detector of the Pierre Auger Observatory, NIM A 620 (2-3) 227-251, 2010

[5] P. Abreu et al., The Pierre Auger Observatory V: Enhancements, in Proc. $32^{\text {nd }}$ ICRC, 2011 , [astro-ph.IM/1107.4807]

[6] The Pierre Auger Collaboration, Contributions to the $33^{\text {rd }}$ International Cosmic Ray Conference, all the contributions for the $33^{r d}$ of the Auger Collaboration are in this paper. [astro-ph.HE/1307.5059]

[7] A. Letessier-Selvon, Highlights from the Pierre Auger Observtory, in Proc. $33^{\text {rd }}$ ICRC, 2013, [astro-ph.HE/1310.4620]

[8] R. Bonino et al. Astrophys. J. 67 (2011) 738

[9] P. Mészáros, Gamma-ray bursts, Rep. Prog. Phys. 69 (8) 2259-2321, 2006

[10] D. Allard et al., Use of water-Cherenkov detectors to detect Gamma Ray Bursts at the Large Aperture GRB Observatory (LAGO), NIMA 595 (1) 70-72, Sep. 2008 and D. Allard et al.. The Large Aperture GRB Observatory in Proc. $31^{\text {st }}$ ICRC, 2009, [astro-ph.HE/0906.0816]

[11] D. Allard et al., Operating Water Cherenkov Detectors in high altitude sites for the Large Aperture GRB Observatory, in Proc. $31^{\text {th }}$ ICRC, 2009.

[12] S. Vernetto, Detection of gamma-ray bursts in the $1 \mathrm{GeV}-1 \mathrm{TeV}$ energy range by ground-based experiments. Astroparticle Physics, 13 (1), 75-86, 2000, [astro-ph.HE/990.4324].

[13] The Pierre Auger Collaboration, The Pierre Auger Observatory scaler mode for the study of solar activity modulation of galactic cosmic rays, JINST 6 (1) P01003, 2011.

[14] H. Asorey, for the LAGO collaboration, The LAGO Solar Project, in Proc. $33^{\text {rd }}$ ICRC, 2013.

[15] The LAGO Collaboration, in preparation, 2014.

[16] H. Asorey, personal communication.

[17] M. Mostafa, The High Altitude Water Cherenkov Observatory, in Proc. $33^{\text {rd }}$ ICRC, 2013, [astro-ph.HE/1310.7237].

[18] H. Asorey and L. Núñez, in Proc. of XIV Latin American Regional IAU meeting, 2013.

[19] N. Smith, talk given at the $12^{\text {th }}$ Topics in Astroparticle and Underground Physics (TAUP11), 2011, Munich, Germany, the slides are available at http://taup2011.mpp.mpg.de/.

[20] X. Bertou, The ANDES Underground Laboratory, in Proc. 33 ${ }^{\text {rd }}$ ICRC, 2013, [astro-ph.IM/1308.0059] and X. Bertou, European Phys. Journal Plus 127 (9) pp 104-108, 2012.

[21] P. A. N. Machado, T. Mühlbeier, H. Nunokawa, R. Zukanovich Funchal, Potential of a Neutrino Detector in the ANDES Underground Laboratory for Geophysics and Astrophysics of Neutrinos, Phys. Rev. D 86, 125001, 2012, [astro-ph.HE/1207.5454].

[22] See andeslab.org and andeslab.org/cles.php. 